\title{
LES ÉLÉMENTS SUPERLOURDS
}

\author{
M. EPHERRE (*) \\ C. STEPHAN $(* *)$
}

\begin{abstract}
Résumé. - L'état actuel de la recherche des éléments superlourds est présenté. Après un bref rappel des prévisions théoriques concernant leur stabilité les problèmes rencontrés pour réaliser leur synthèse en laboratoire, et les espoirs que font naître les futurs accélérateurs d'ions lourds sont exposés. Les contraintes imposées à la fois par la physique nucléaire et l'astrophysique à leur existence dans la nature sont évoquées, et les techniques très sensiblès mises au point au cours des 2 dernières années pour leur détection sont décrites. Les résultats négatifs obtenus jusqu'à maintenant ne paraissent néanmoins pas définitifs.
\end{abstract}

\begin{abstract}
The present status of the search for super heavy elements is presented. Theoretical predictions indicate that superheavy elements (around $Z=114, \mathrm{~A}=298,301$ ) would have sufficient stability to allow their identification, but no suitable nuclear reactions have been found for their synthesis. Improvements could be achieved with the new heavy ions accelerators. The nucleosynthesis of the superheavy elements in nature seems difficult, not impossible, and very sensitive techniques have been developped in the last 2 years for their detection. Despite the negative results obtained until now any final conclusions about their existence would be premature.
\end{abstract}

1. Introduction. - Il y a environ 40 ans qu'est apparue pour la première fois la possibilité d'existence de noyaux stables au-delà de la péninsule de matière nucléaire. Mais c'est en 1966 que les calculs de Myers et Swiatecki [1] la formulent de façon quantitative et suggèrent la localisation de l'îlot de stabilité autour de $Z=114, N=184$. A partir de 1968, un grand nombre de théoriciens à travers le monde s'intéressent au problème. Les calculs se perfectionnent en gardant généralement comme point de départ la superposition à un modèle macroscopique qui tient compte des variations moyennes de l'énergie potentielle nucléaire, d'une description microscopique des effets locaux à l'aide d'un modèle à particules indépendantes, méthode développée particulièrement par Strutinsky [2]. Cependant, les développements récents les plus intéressants ont été obtenus par l'application de la méthode Hartree-Fock qui utilise l'interaction nucléon-nucléon de type Skyrme [3]. Ces calculs, dans la région des actinides comme dans celle des superlourds, reproduisent bien, en particulier, la barrière de fission à 2 maxima. Ils confirment les plus importants résultats théoriques antérieurs : plus grande stabilité des couches $Z=114, N=184$, avec comme meilleur candidat pour la stabilité les éléments ${ }^{294} 110$ et ${ }^{296} 112$. Néanmoins, dans ces calculs les barrières de fission se trouvent abaissées de 2 à $3 \mathrm{MeV}$ (à cause d'une corrélation neutron-proton qui conduit à une réduction de l'énergie coulombienne et qui n'existe pas dans les calculs de type Strutinsky) ce qui entraîne pour les noyaux les plus stables $\left({ }^{294} 110\right)$ un abaisse-

(*) Laboratoire René-Bernas, CSNSM, Orsay, France.

(**) Institut de Physique Nucléaire, Orsay, France. ment de la durée de vie d'un facteur $10^{8}$. Or les calculs de Nilsson et ses collaborateurs [4] en 1968 laissaient prévoir pour les noyaux les plus stables une durée de vie de l'ordre de $10^{8}$ ans ce qui avait stimulé la recherche des éléments superlourds dans la nature. Les prévisions actuellement en faveur indiquent une durée de vie maximale de $10^{4}$ ans. Toutefois, il ne faut pas oublier que beaucoup de paramètres utilisés dans les calculs ne sont pas très sûrs et qu'une très faible erreur entraîne des variations de plusieurs ordres de grandeur des durées de vie. Si certains expérimentateurs qui avaient entrepris la recherche des éléments superlourds dans la nature pensent maintenant qu'ils n'existent probablement pas sur terre en quantité mesurable, d'autres cependant continuent de poursuivre les recherches. En ce qui concerne leur synthèse en laboratoire par des réactions avec ions lourds, elle paraît toujours possible et beaucoup d'espoirs sont fondés sur le développement de puissants accélérateurs à Doubna, Berkeley, Darmstadt et Caen.

2. Tentatives de synthèse en laboratoire. - Tous les efforts accomplis ces dernières années pour synthétiser les éléments superlourds se sont traduits par des échecs.

Les premières expériences ont tenté de réaliser la synthèse de nouveaux éléments transuraniens par irradiation d'uranium ou d'éléments plus lourds avec des flux intenses de neutrons, d'abord dans des réacteurs, puis en 1966-1967, à l'aide d'explosions nucléaires souterraines. Cette voie qui permit la synthèse des éléments jusqu'à $Z=100$, dut être abandonnée, les durées de vie des éléments créés 
devenant de plus en plus faibles (Fig. 1). Le flux de neutrons était insuffisant pour que des noyaux très riches en neutrons soient atteints avant de se désintégrer. On reviendra plus loin sur ce mécanisme.

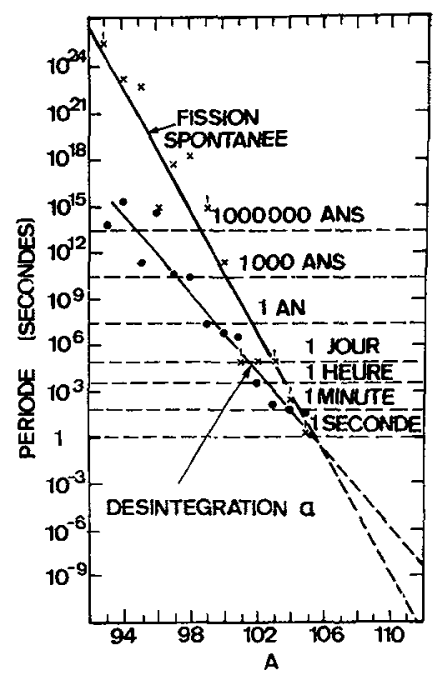

FIG. 1. - Durées de vie les plus longues des éléments transuranıens connus (d'après G. T. Seaborg [18]).

Les recherches se sont alors orientées vers les réactions de fusion avec ions lourds. Les premiers essais furent réalisés à Berkeley avec la réaction ${ }^{248} \mathrm{Cm}+{ }^{40} \mathrm{Ar}$. Dans la mesure où le produit final correspondant à la fusion complète ${ }_{114}^{288} \mathrm{X}_{174}$ se situait loin du nombre magique $N=184$, l'échec ne fut pas une surprise.

Dans les années 1970-71, les expériences menées à Orsay avec des ions $\mathrm{Kr}$ - accélérés par Alice [5] et à Dubna avec des ions $\mathrm{Zn}$ - obtenus à l'aide du cyclotron Y-300 [6] - éveillèrent un vif intérêt. Le groupe d'Orsay, avec une cible de ${ }^{232} \mathrm{Th}$, pouvait espérer former des noyaux jusqu'à $Z \leqslant 126$ et $N \simeq 184$. Ces expériences permirent seulement d'établir la limite supérieure de la section efficace de formation des noyaux superlourds dans cette réaction soit $10^{-30} \mathrm{~cm}^{2}$. A Dubna, des cibles de ${ }^{238} \mathrm{U}$ et ${ }^{243} \mathrm{Am}$ ont été bombardées avec des ions ${ }^{66,68} \mathrm{Zn}$, ce qui permettait, en principe, de synthétiser des noyaux superlourds de $Z=120-125$ et $N$ voisin de 184 . Ces expériences, une fois de plus, n'aboutirent qu'à donner une limite supérieure de section efficace de formation de $5 \times 10^{-32} \mathrm{~cm}^{2}$. Cette méthode avait pourtant déjà permis de synthétiser un certain nombre d'isotopes d'éléments de $Z=100$ à $Z=105$.

Ces expériences ont néanmoins ouvert un nouveau domaine d'étude de l'interaction entre ions lourds, celui de la macrophysique nucléaire qui s'avère particulièrement intéressant. On a pu constater en particulier, grâce aux premières expériences, que la barrière de réaction pour la fusion de 2 noyaux, de masse 100 environ, est bien supérieure à celle prévue par les calculs classiques [7]. On a observé également que la réaction dans laquelle les produits sont assez voisins du projectile et du noyau cible - avec une énergie cinétique correspondant à l'énergie coulombienne de répulsion de deux fragments de fission ordinaire représente une fraction considérable de la section efficace totale. Ces réactions de transfert ayant des sections efficaces particulièrement importantes, on a pensé les utiliser pour produire des noyaux superlourds, par apport d'un nombre important de nucléons au noyau-cible. Les résultats obtenus à Orsay, concernant la distribution des fragments lors de la réaction $\mathrm{Ar}+\mathrm{Th}$, ne sont cependant pas encourageants [8]. Le fragment lourd semble emporter la plus grande partie de l'énergie d'excitation, ce qui augmente considérablement la probabilité de fission. D'autre part, le rapport $N / Z$ tend à s'équilibrer entre les deux noyaux en interaction. Le fragment lourd aura donc tendance à céder des neutrons à l'ion léger. Le noyau superlourd sera donc formé loin de l'îlot de stabilité. Ce dernier effet peut cependant ne pas intervenir si on bombarde la cible lourde par un ion déjà très riche en neutrons. Ceci a été tenté en bombardant des cibles lourdes avec des protons très énergiques. Les fragments obtenus par éclatement du noyau devaient alors bombarder à leur tour les noyaux cibles. Ces réactions secondaires se sont avérées trop peu probables avec des protons pour pouvoir aboutir [9]. Ce peut être par contre une voie intéressante avec des ions lourds. Les fragments légers riches en neutrons, fabriqués par transfert, pourraient induire une réaction secondaire sur le noyau cible. En attendant des faisceaux d'ions lourds intenses qui permettraient de réaliser cette expérience, on met' quelque espoir dans la réaction

$$
{ }^{48} \mathrm{Ca}+{ }^{250} \mathrm{Cm} \rightarrow{ }_{116}^{198} \mathrm{X} \stackrel{\alpha}{\rightarrow}{ }_{114}^{294} \mathrm{X},
$$

le noyau final étant très près du centre de l'îlot de stabilité calculé.

3. Possibilités de synthèse dans la nature. - Il est généralement bien admis que les éléments plus lourds que le plomb, présents dans la nature, ont été formés par un mécanisme de capture rapide de neutrons (processus r). La question est de savoir si cette capture pourrait se poursuivre assez loin pour atteindre l'îlot de stabilité. Beaucoup de réponses différentes ont été données en particulier parce que les contraintes imposées par la physique nucléaire doivent être satisfaites dans un contexte astrophysique.

Néanmoins, avec le temps, les modèles deviennent plus réalistes, les calculs se perfectionnent et nous allons essayer de rendre compte de la situation actuelle.

$\mathrm{Du}$ point de vue de l'astrophysique un milieu à très forte densité de neutrons est nécessaire pour que la capture puisse se faire à un rythme rapide : une supernova en explosion paraît être un bon site. Des calculs hydrodynamiques permettent de suivre en fonction du temps, échauffement, refroidissement, compression, expansion de chaque morceau de l'étoile en explosion. La densité de neutrons ( $n$ ) et la tempé- 
rature $(T)$ vont déterminer le trajet suivi par le processus r (Fig. 2), et de leurs valeurs au moment de la trempe des réactions nucléaires, c'est-à-dire de leur arrêt brutal pendant la phase de refroidissement va dépendre le point d'arrêt du trajet. En effet, s'il y a capture rapide de neutrons à partir d'un noyau stable ( ${ }^{56} \mathrm{Fe}$ par exemple Fig. 2) le nombre de masse $A$ augmente à $Z$ constant $\left(t_{\beta} \gg t_{(\mathrm{n} \gamma)}-t_{\beta}\right.$ durée de vie par rapport à l'émissión $\beta$, etc...) jusqu'au noyau pour lequel s'établit l'équilibre $(\mathrm{n} \gamma) \leftrightarrows(\gamma \mathrm{n})$. Il y a alors décroissance $\beta ; Z \rightarrow Z+1$ puis de nouveau capture de neutrons, et ainsi de suite. Le trajet se tient bien à droite de la vallée de stabilité. Quand ure couche magique à $N$ neutrons est atteinte, la trajectoire remonte vers la vallée - le $(N+1)$-ième neutron étant très peu lié - jusqu'à ce que $t_{\beta}$ devienne assez grand pour permettre l'addition de ce $N+1$-ième neutron. Ces isotopes à nombre magique de neutrons sont des isotopes d'attente qui après décroissance $\beta$ donneront des pics d'abondance autour de nombres de masse $A$ un peu plus faibles que ceux des éléments stables ayant le même nombre de neutrons $(A=80$, 130, 194).

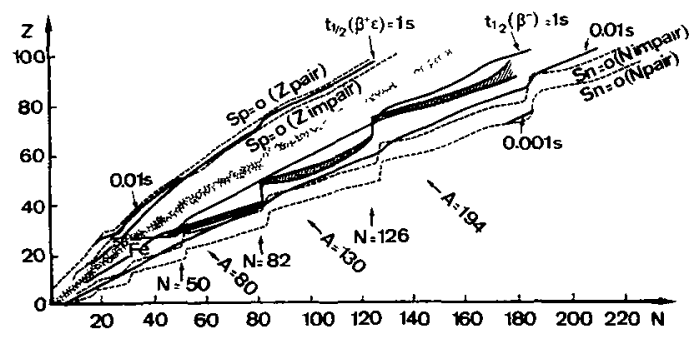

Fig. 2. - Un exemple de trajet du processus $r$ (d'après E. R. Hilf et al. [18]).

Cette trajectoire $r$ se poursuit jusqu'au moment où la fission induite par neutrons arrête le processus de capture $t_{\mathrm{n} y} \gg t_{\mathrm{nf}}$. La possibilité de former des éléments superlourds dépend de la réponse que l'on peut donner aux 2 questions suivantes :

1) La fission induite par les neutrons peut-elle stopper le processus de capture avant que la région des masses $A=300$ ne soit atteinte ?

2) $\mathrm{Si}$ ces noyaux très riches en neutrons sont produits peuvent-ils pendant la phase de refroidissement décroître vers la vallée de stabilité sans subir la fission spontanée?

Pour tenter de répondre à la première question, on utilise les calculs de barrières de fission et d'énergie de séparation de neutrons développés dans la région des éléments superlourds. Sur la figure 3-due à Howard et Nix [10] - basée sur les résultats les plus récents, sont portées en fonction de $N$ et $Z$ les énergies minimales de neutrons pouvant induire la fission. On conclut en général que le dernier noyau qu'il est possible de former par captures successives de neutrons est le noyau $Z=96, N=186$.

Cependant, ainsi que l'a fait remarquer O. Johns [11], le calcul a probablement été limité à une zone trop restreinte en $Z$ et $N$. Il a, en effet, remis en question le trajet unique dans le plan $(N, Z)$ du processus $r$ proposé par Seeger, Fowler et Clayton en 1965 dans leur modèle quasi statique de supernovae, pour reproduire les pics d'abondance des éléments de masse 130 et 194 [12]. Les abondances des éléments lourds pourraient être le résultat de différents trajets du processus $r$, conséquences d'évolutions différentes des explosions de supernovae, ce que les modèles dynamiques laissent prévoir, à cause de conditions initiales variables.

Chaque trajet est caractérisé par un paramètre $\gamma$ fonction uniquement de $T$ et $n$. Ceux qui traversent la portion du plan $(N, Z)$ représenté sur la figure 3 correspondent à des valeurs $4,3<\gamma<1,4$; or, il est possible qu'au moment de la trempe $\gamma$ soit voisin de 1 ou $0,5 \mathrm{MeV}$ (ce qui supposerait pour la supernovae un taux d'expansion $a=1 / 200$ ou $1 / 500$ ) et il serait intéressant de savoir où le trajet correspondant à $\gamma=0,5$ intercepte la région $E_{\mathrm{S}}<1 \mathrm{MeV}$.

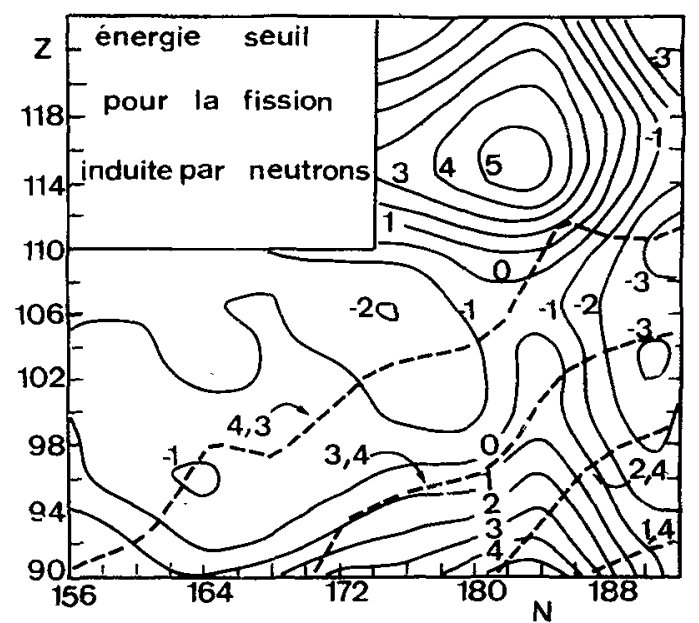

FIG. 3. - En trait plein contours des énergies-seull pour la fission induite par neutrons d'après [10]. En pointillé contours du paramètre gamma caractérisant chaque trajet du processus $r$ suivant [11].

Il resterait alors à répondre à la $2^{\mathrm{e}}$ question : l'îlot de stabilité peut-il être atteint bien que la chaîne de désintégration $\beta$ ait à traverser la région autour de $Z=100, N=190$ où les temps de vie par rapport à la fission spontanée sont de l'ordre de $10^{-21}$ ans au moins?

Bien qu'on ne puisse pas encore répondre à ces 2 questions, il semble néanmoins prématuré d'infirmer toute possibilité de nucléosynthèse d'éléments superlourds.

4. Recherches dans la nature. - A cause de l'incertitude des prévisions, on ne s'est pas limité à rechercher seulement ceux des éléments prévus avec une longue durée de vie, mais les éléments de l'îlot dans leur ensemble [13].

OÙ LES RECHERCHER ? - Le choix de l'échantillonnage est essentiel. En général, on a supposé que ces éléments se comporteraient dans les processus 
chimiques et géologiques comme.leurs homologues plus légers et ce sont des matériaux contenant les éléments de la dernière ligne du tableau périodique qui ont été le plus souvent étudiés. Néanmoins, il peut en être autrement si, par exemple, les éléments superlourds ont des rayons ioniques différents ou préferrent des états de valence plus faibles que ceux de leurs homologues [14].

Selon les résultats publiés, plusieurs centaines d'échantillons ont été examinés parmi lesquels des minerais de platine, d'or, de plomb, leurs produits et sous-produits de raffinage, du platine à l'état natif, des verres au plomb. On les a également recherchés dans des échantillons extra-terrestres : roches rapportées de la lune, météorites, rayonnement cosmique. On a également pensé que les éléments superlourds du rayonnement cosmique qui auraient atteint la terre devraient se trouver concentrés dans les sédiments marins et on les a recherchés dans des nodules de manganèse - bons pièges pour la plupart des métaux lourds - extraits par $5000 \mathrm{~m}$ de fond dans l'océan Pacifique.

COMMENT Les MetTRe EN ÉvidenCe ? - Le plus souvent, on a recherché les événements de fission spontanée. En effet, la fission spontanée étant très rare parmi les radio-éléments naturels connus, la détection des événements de fission peut se faire sans traitement chimique des échantillons. En recherchant ces fissions spontanées, on fait l'hypothèse, étayée par les calculs théoriques, que même si les éléments superlourds se désintègrent principalement suivant un autre mode, ils ont au moins des descendants qui fissionnent. Principalement, 2 méthodes ont été utilisées pour rechercher les fissions spontanées :

a) L'analyse de traces ou défauts que les fragments de fission créent dans des solides isolants tels que verre, mica, plastique. Les traces dues aux éléments superlourds seraient plus longues, leurs fragments de fission ayant plus d'énergie cinétique.

b) Le comptage des neutrons qui accompagnent chaque événement de fission. La théorie prévoit en effet que la fission d'un noyau superlourd s'accompagne de l'émission d'un plus grand nombre de neutrons que celle de l'uranium. Les résultats n'ont pas toujours été entièrement négatifs, des indices sont apparus quelquefois, anéantis par des recherches complémentaires. Ainsi les traces de fission spontanée observées dans des verres au plomb par l'équipe du professeur Flerov se sont avérées être par la suite des traces de fission induite dans le plomb par le rayönnement cosmique; rayonnement cosmique également responsable du taux de comptage de neutrons bien au-dessus du bruit de fond qu'ont observé Hermann et ses collaborateurs dans des échantillons métalliques (neutrons résultant de la spallation des métaux par le rayonnement cosmique).
Dans certaines météorites, les plus primitives chondrites carbonées, la composition isotopique du xénon de fission ne correspond à aucune de celles connues pour la fission des actinides et Anders et Larimer [15] ont suggéré sur la base de considérations physicochimiques que ce xénon pourrait provenir de la fission d'éléments de $Z=111$ ou 115. Dans le rayonnement cosmique même, P. H. Fowler et ses collaborateurs avaient observé un événement de charge $Z=105 \pm 3$ mais les derniers résultats dans ce domaine ne sont toujours pas encourageants. Ce sont ceux des expériences préparées à bord du vaisseau spatial Skylab. $\mathrm{Au}$ total, $1,2 \mathrm{~m}^{2}$ de détecteur (Lexan) a été exposé pendant $253 \mathrm{j}$ au flux de rayonnement cosmique, ce qui a permis en particulier de doubler, par cette seule expérience, les statistiques mondiales d'observation de noyaux $Z>70$. Aucun noyau de $Z \geqslant 110$ n'a été observé sur les 112 événements dépouillés (Fig. 4). En regroupant toutes les observations faites en ballon et à bord de Skylab, on peut maintenant indiquer comme limite d'abondance relative des éléments superlourds dans le rayonnement cosmique [16]

$$
\frac{[Z \gtrsim 110]}{[70 \leqslant Z \leqslant 83]}<0,006
$$

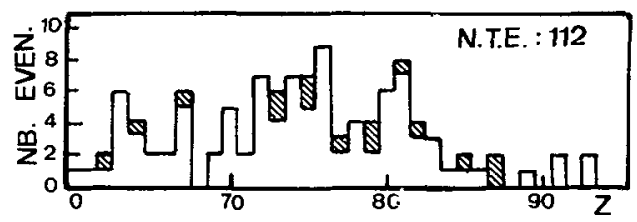

FIG. 4. - Distribution des 112 événements du rayonnement cosmique observés à bord de Skylab d'après [15] les zones hachurées indiquent des résultats pouvant être éventuellement révisés.

ce qui correspond à un flux inférieur à 0,3 noyau $/ \mathrm{m}^{2}$ sr.an. Peut-être serait-il plus intéressant de retourner à l'étude des traces fossiles dans les météorites : quelques $\mathrm{cm}^{2}$ de cristal exposé pendant une durée standard de $10^{7}$ ans représentent une exposition de plus de $10^{5} \mathrm{~m}^{2}$ sr.jour de détecteur. Mais il faudrait, pour cela, développer une méthode sûre d'identification de charges des traces individuelles.

Les résultats négatifs obtenus dans l'étude des échantillons terrestres peuvent paraitre moins décourageants en ce sens qu'ils sont la conséquence du double choix de l'échantillonnage et de la méthode de détection; choix qui est peut-être à revoir si comme nous l'avons mentionné précédemment les éléments superlourds ne suivent pas leurs homologues plus légers dans les processus chimiques et géologiques.

EXPÉRIENCE ORSAY-SWIERK. - Les expériences sur les échantillons terrestres mentionnées précédemment spéculaient sur les propriétés calculées de la fission spontanée, une méthode très différente a été mise au point à Orsay [17]. Elle consiste à isoler les éléments superlourds qui pourraient exister dans l'échantillon étudié, à l'aide du séparateur électromagnétique d'isotopes Sidonie, puis à les mettre en évidence par 
la fission induite par neutrons. Les calculs de barrières de fission prévoient des valeurs allant de 9 à $12 \mathrm{MeV}$. Les énergies de séparation de neutrons devraient être de l'ordre de 3 à $4 \mathrm{MeV}$. Les neutrons de fission de l'uranium présents dans un réacteur piscine devraient induire la fission des noyaux superlourds. Cette méthode présente plusieurs avantages sur les précédentes :

La séparation préalable permet d'isoler les noyaux superlourds éventuels de l'uranium et du thorium toujours présents dans les échantillons naturels et qui subissent également la fission par neutrons de pile. L'utilisation de la fission induite pour mettre en évidence les noyaux superlourds au lieu d'étudier la fission spontanée permet d'avoir une réponse immédiate. En effet, en supposant une section efficace de fission de l'ordre du barn, on obtient dans le réacteur autant de fissions en quelques jours qu'en plusieurs années avec la fission spontanée. Les détecteurs de fragments de fission sont des lamelles de quartz capables de supporter des flux très intenses de neutrons. La limite actuelle de détection de cette méthode se situe au niveau de $10^{-15} \mathrm{~g} / \mathrm{g}$. Des fissions ont effectivement été observées dans la région des masses 300 , pour quelques échantillons où d'autres auteurs avaient cru voir des indices intéressants. Cependant, on ne peut pas exclure la possibilité que les masses observées soient en fait des molécules contenant de l'uranium ou du thorium. Pour savoir s'il ne s'agissait effectivement que d'une contamination, les masses collectées ont été irradiées d'abord en interposant un écran de cadmium sur le trajet des neutrons, puis sans écran de cadmium. Les sections efficaces de fission étant connues dans chaque cas pour les isotopes ${ }^{235} \mathrm{U},{ }^{238} \mathrm{U},{ }^{232} \mathrm{Th}$, on devait pouvoir éliminer ainsi les fissions dues à une contamination. Le fait que plusieurs des masses collectées ne se comportaient pas vis-à-vis de la fission comme les isotopes ci-dessus constituait un indice intéressant; mais néanmoins insuffisant. Une expérience complémentaire a été alors réalisée [19]. Si des composés moléculaires sont formés temporairement dans le séparateur d'isotopes, ils doivent être détruits au moment de l'impact sur le collecteur. Les masses ont donc été collectées pour moitié sur des quartz, moitié sur des rubans de rhénium. Dans les premiers, on recherche les fissions. Les seconds en regard desquels des fissions sont observées sont placés à la source d'ion d'un spectromètre de masse. L'émission thermo-ionique des ions implantés dans le rhénium permet de détecter la présence de $10^{7}$ atomes d'uranium. Les résultats obtenus pour un échantillon de platine sont présentés sur la figure 5 . On constate qu'à chaque masse pour laquelle des fissions ont été observées, on trouve de l'uranium ou du thorium dans les filaments de rhénium analysés. Il y a donc contamination. Il reste cependant que les rapports isotopiques ${ }^{235} U,{ }^{238} U$ que l'on obtient ne correspondent pas à ceux que l'on observe dans la nature. Ce phénomène n'a pour l'instant pas été expliqué.

Nous envisageons d'améliorer encore la sensibilité de la méthode en effectuant une concentration chimique préalable. On peut espérer ainsi gagner un facteur 1000 en sensibilité. Le gain sera d'autant plus grand que la fraction uranium + thorium sera mieux séparée du reste de l'échantillon.
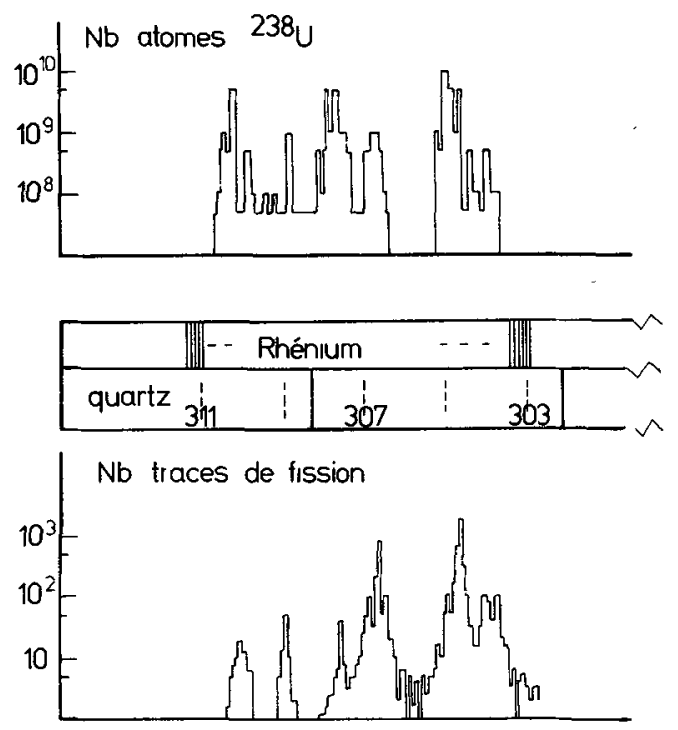

FIG. 5. - Résultats montrant la corrélation entre le nombre de traces de fission induites observées dans le quartz et le nombre d'atomes d'uranium mesurés sur les filaments de rhénium entre les masses 303 et 311 .

5. Conclusion. - Alors que tous les calculs théoriques qui se sont perfectionnés depuis plusieurs années continuent de conclure à l'existence d'un îlot de stabilité, la quête des éléments superlourds pour les expérimentateurs persiste à rester négative. Peutêtre que dans les prochaines années, la mise en service de nouveaux accélérateurs à ions lourds permettra enfin d'en réaliser la synthèse. Leur recherche a cependant ouvert la voie à ce qu'on appelle maintenant la macrophysique nucléaire qui se révèle très intéressante pour l'étude de la matière nucléaire. $\mathrm{La}$ méthode mise au point à Orsay pour essayer de découvrir les noyaux superlourds dans la matière terrestre devrait permettre sinon de démontrer leur existence, au moins de recueillir des indices sérieux. La découverte d'éléments superlourds serait pourtant très importante. Elle permettrait de raffiner les modèles nucléaires en raison des extrapolations lointaines que ces éléments exigent. Leur présence dans la nature permettrait de recueillir des informations plus précises sur les supernovae, si elles sont effectivement le site du processus $\mathrm{r}$. Ce domaine a été, pendant plusieurs années, le centre d'intérêt de centaines de physiciens et malgré l'absence totale d'indices a donné lieu à un nombre considérable de travaux. 


\section{Bibliographie}

[1] Myers, W. D., Swiatecki, W. J., Nucl. Phys. 81 (1966) 1.

[2] Strutinsky, V. M., Nucl. Phys. A 122 (1968) 1.

[3] Beiner, M., Flocard, H., Veneroni, M., Quentin, P., Phys. Scripta 10A (1974) 84.

[4] Nilsson, S. G., Tsang, C. F., Sobiczewski, A., Szymanski, Z., Wycek, S., Gustafson, G., Lamm, I., Moller, P., NiLsson, B., Nucl. Phys. A 131 (1969) 1.

[5] Colombani, P., Gatty, B., Jacmart, J. C., Lefort, M., Peter, J., Riou, M., Stephan, C., Tarrago, X., Phys. Lett. 42B (1972) 208.

[6] Demin, A. G., Kusch, W., Mrller, M. B., Passiuk, A. S., Pleve, A. A., Tetiakov, V. D., Proc. Inter. Conf. Heavy Ion Physics, Dubna (1971), JINR, D 7-5769, p. 169.

[7] Lefort, M., Phys. Rev. C 12 (1975) 686.

[8] Jacmart, J. C., Frascaria, N., Poffe, N., Colombani, P., Doubre, H. M., Riou, M., Roynette, J. C., StePhan, C., Nucl. Phys. A 242 (1975) 175.

[9] Westgaard, L., Erdal, B. R., Hansen, P. G., Kugler, E., Sletten, G., Sundell, S., Frisch, T., Henrich, E.,
Theis, W., Wolf, G. K., Camplan, J., Klapisch, R., Meunier, R., Poskanzer, A. M., Stephan, C., Tys, J., Nucl. Phys. A 192 (1972) 517.

[10] Howard, W. M., NIX, J. R., Nature 247 (1974) 17. Howard, W. M., Phys. Scripta 10A (1974) 138.

[11] JoHNs, O., Rapport LRB 74/3, Orsay (1974).

[12] Seeger, P. A., Fowler, W. A., Clayton, D. D., Ap. I. Suppl. 11 (1965) 121.

[13] Hermann, G., Phys. Scripta 10A (1974) 71.

[14] Keller, O. L. Jr, Nestor, C. W. Jr, Carlson, T. A., Fricke, B., J. Phys. \& Chem. solids 77 (1973) 1806.

[15] Anders, E., Larimer, J. W., Science 175 (1972) 981.

[16] Israel, M. H., Price, P. B., Waddington, C. J., Physics Today 28 (1975) 23.

[17] Stephan, C., Tys, J., Sowinski, M., Cieslak, E., Meunier, M., J. Physique 36 (1975) 105.

[18] Proceedings of Nobel Symposium 27 on Superheavy Elements, Ronneby, Sweden, juin 1974, Phys. Scripta 10A (1974).

[19] EPHerre, M., KLAPISCH, R., to be published in Phys. Rev. C, jan. 1976 issue. 